BULLETIN OF THE

AMERICAN MATHEMATICAL SOCIETY

Volume 81, Number 1, January 1975

\title{
JOSEPH L. WALSH \\ IN MEMORIAM
}

\author{
BY MORRIS MARDEN
}

Threescore years and ten is the biblical measure of a man's normal life span. Yet in modern times his surpassing of this bound is not unusual. However, it becomes noteworthy when he is blessed with undiminished physical and mental vigor. This was true of Joe Walsh. "His spirit was marvelous until the end, and he spoke with gratitude for his many productive years." 1

Joseph Leonard Walsh died on December 10,1973 at the age of seventy eight years in his home at University Park, Maryland. This site is not far from where he was born on September 21, 1895, as son of Reverend and Mrs. John Leonard Walsh. Most of his academic life as student, teacher and scholar was spent at Harvard University. In 1916 Harvard awarded him the S.B. degree, summa cum laude, and at the same time a Sheldon Traveling Fellowship for study at the Universities of Chicago and Wisconsin. On his return to Harvard in 1917, Walsh began some studies under Maxime Bôcher, but their progress was interrupted by World War I and his enlistment as an ensign in the U.S. Navy. In 1920 Harvard granted him a Ph.D. and also a second Sheldon Traveling Fellowship, this time for study in Paris under Paul Montel. Back from Europe in 1921 he joined the Harvard faculty, but in 1925 took a leave-of-absence for a year's research at Munich under Carathéodory. Returning again to Harvard, he was promoted through the ranks to a full professorship in 1935 and served as department chairman from 1937 to 1942. In the latter year he was recalled to active duty in the U.S. Navy as a lieutenant commander. When he returned to Harvard in 1946, he was appointed to the prestigous Perkins Professorship, which he held until his retirement in 1966. A semester earlier he began a research professorship at the University of Maryland, in which position he remained fully active, working with Ph.D. and post doctoral students, until a few months before his death.

During Walsh's lifetime he received many academic and nonacademic honors. Among them was his election in 1936 to the National Academy of Science, and in 1937 to the vice-presidency of the American Mathematical Society. He was elected for a two-year term as president of the Society in 1949. This was a crucial period for the Society when it was experiencing

\footnotetext{
${ }^{1}$ Letter from Mrs. Joseph L. Walsh.
} 


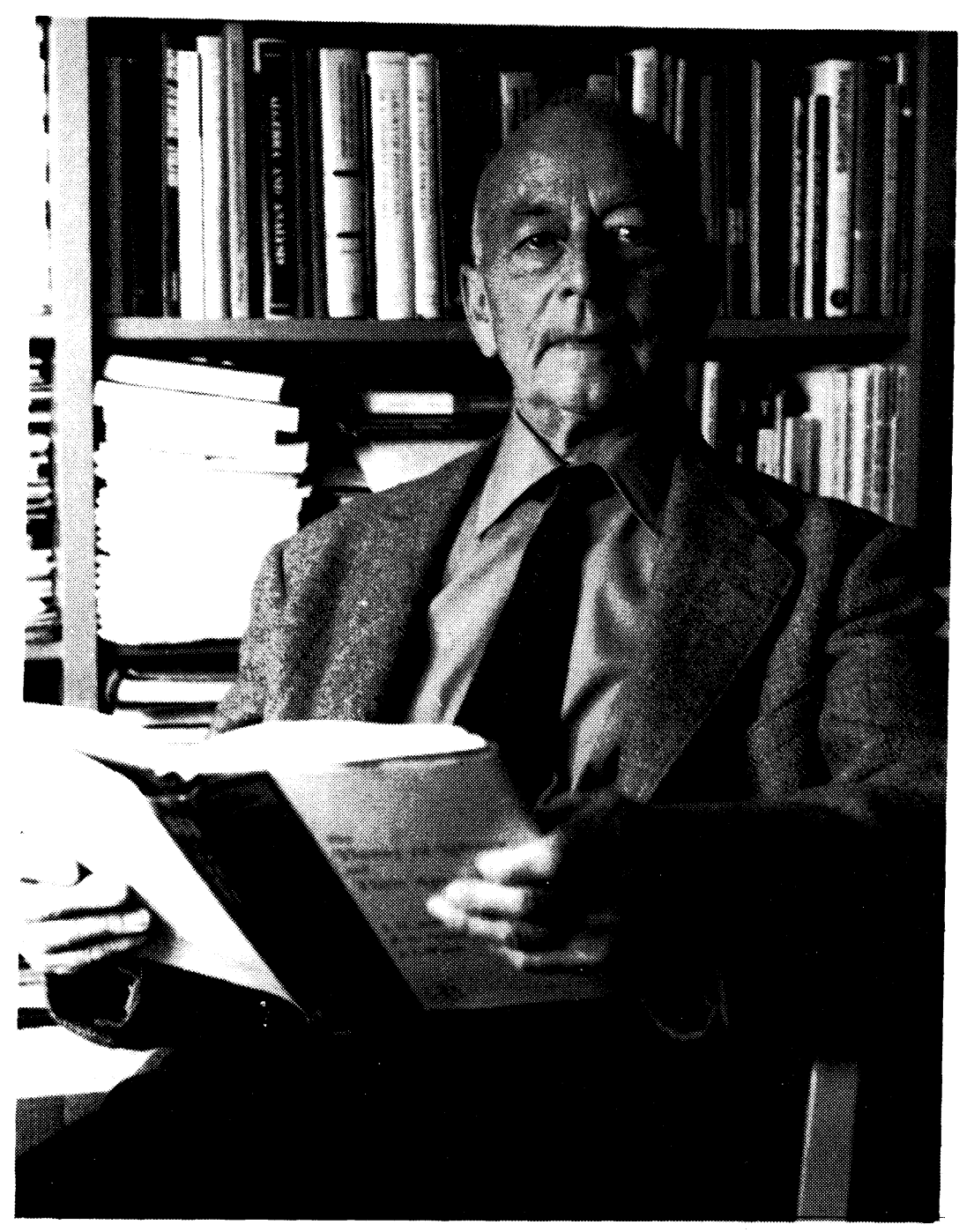

JOSEPH L. WALSH (1895-1973) 
growing pains due to a rapid increase in membership and in research publications. It was the period in which the Society created the post of Executive Director and moved its headquarters to Providence. Also during this period Walsh served as chairman of the organizing committee for the International Mathematical Congress, held in Cambridge during August 1950, the first since World War II. At about this time Walsh was recipient of a nonmathematical honor-promotion to the rank of captain in the U.S. Naval Reserve. In his later years Walsh was twice honored by the dedication of volumes of mathematical journals: SIAM J. (2) 3 (1966) on his seventieth birthday in 1965, and J. Approximation Theory 5 (1972) on his seventy-fifth birthday in 1970.

These mathematical honors were well deserved in view of the quantity and quality of his original research. Starting with his first publication in 1916, while still an undergraduate, he wrote, singly or jointly with students and others, a total of 279 research, expository, and book review articles as well as seven books. Though these papers covered a wide range of topics, they were, broadly speaking, concerned with four general areas:

(I) The relative location of the zeros of pairs of rational functions such as a polynomial and its derivative.

(II) Zeros and topology of extremal polynomials.

(III) The critical points and level lines of Green's function and other harmonic functions.

(IV) Interpolation and approximation of continuous, analytic, or harmonic functions.

Regarding the general area (I), this was Walsh's first main research interest. His doctoral thesis was entitled On the roots of the jacobian of two binary forms. It was written under the guidance of Maxime Bôcher who had proved that if $F$ and $G$ are binary forms of the same degree and if all the zeros $a_{j}$ of $F$ lie in a circular region $A$ and all the zeros $b_{j}$ of $G$ lie in a circular region $B$ with $B \cap A=\varnothing$, then all the zeros $c_{k}$ of the jacobian $J(F, G)$ lie in $A \cup B$. Like Bôcher, Walsh used geometric and physical methods, interpreting the $c_{k}$ as equilibrium points in the field due to positive masses at the points $a_{j}$ and negative masses at the points $b_{j}$ with an inverse distance force law. Walsh's results are generalizations of the Lucas theorem that the convex hull of the zeros of a polynomial $f$ contains all the critical points of $f$. These results are described in Walsh's papers and in M. Marden, Geometry of polynomials, 2nd ed., Math Surveys, no. 3, Amer. Math. Soc., Providence, R.I., 1966, MR 37 \#1562. The most striking of these results are the following three:

(1) If an $n$th degree polynomial $f$ has $n_{1}$ zeros in a disk $\left|z-c_{1}\right| \leqq r_{1}$ and the remaining $n_{2}=n-n_{1}$ zeros in the disk $\left|z-c_{2}\right| \leqq r_{2}$, then any critical 
point of $f$ not in one of these disks lies in a third, "average" disk

$$
\left|z-\left(n_{2} c_{1}+n_{1} c_{2}\right) / n\right| \leqq\left(n_{2} r_{1}+n_{1} r_{2}\right) / n \text {. }
$$

(2) If $C_{1}, C_{2}$ and $C_{3}$ are disjoint circular regions and if a rational function $f$ has in the extended plane all its zeros in $C_{1} \cup C_{2}$ and all its poles in $C_{3}$, then any critical point of $f$ not in $C_{1} \cup C_{2} \cup C_{3}$ lies in a circular region $C_{4}$. The boundary $\partial C_{4}$ of $C_{4}$ is the locus of the point $z_{4}$ defined by the cross ratio $\left(z_{1}, z_{2}, z_{3}, z_{4}\right)=$ const when $z_{1}, z_{2}, z_{3}$ vary independently on the circles $\partial C_{1}, \partial C_{2}, \partial C_{3}$ respectively.

(3) Let the form $\Phi\left(z_{1}, z_{2}, \cdots, z_{n}\right)$ be of degree one in each $z_{j}$, and of total degree $n$ and symmetric in the set $z_{1}, z_{2}, \cdots, z_{n}$. Let $C$ be a circular region containing the $n$ points $z_{j}=z_{j 0}, j=1,2, \cdots, n$. Then in $C$ there exists at least one point $\zeta$ such that $\Phi(\zeta, \zeta, \cdots, \zeta)=\Phi\left(z_{10}, z_{20}, \cdots, z_{n 0}\right)$.

Regarding the general area (II), the methods and results were suggested in part by those in area (I). Given a closed bounded set $E$ containing at least $n+1$ points and the class $P_{n}$ of all polynomials $z^{n}+a_{1} z^{n-1}+\cdots+a_{n}$, an infrapolynomial $p$ on $E$ means a polynomial $p \in P_{n}$ with the property

$$
\max _{z \in E}|p(z)|=\min _{q \in P_{n}} \max _{z \in E}|q(z)| \text {. }
$$

The zeros of $p$ play a role vis-à-vis set $E$ similar to that of the critical points of a polynomial $f$ vis-à-vis the zeros of $f$. For instance, Fejér proved that the zeros of $p$ lie in the convex hull of $E$, and Fekete showed that $p$ satisfies a form involving the points of $E$ that is similar to the form for the logarithmic derivative of $f$ in terms of the zeros of $p$. Walsh explored the subject of infrapolynomials intensively in papers which he published singly or jointly with Fekete, Motzkin, Shisha and Zedek.

Likewise the general area (III) was partly an offshoot of area (I). For example, Walsh proved that if $G$ is the Green's function (with pole at infinity) for an unbounded region $R$ with bounded boundary $B$, then all the critical points of $G$ in $R$ lie in the convex hull of $B$. Thus, the critical points of $G$ play a role similar to the critical points of a polynomial whose zeros lie on $B$. In this connection Walsh also examined in detail the curvature and other characteristics of the level lines of Green's function. These theorems together with their generalizations to harmonic measures and other harmonic functions are developed in Walsh's papers and described in his book, Critical points of analytical and harmonic functions.

As for general area (IV), the subjects of interpolation and approximation encompass about half of Walsh's published articles as well as his now classical treatise entitled Interpolation and approximation. Among his many original results in this area, probably the most important are the 
following two:

(1) Every function continuous on a bounded Jordan arc $J$ can be approximated on $J$ uniformly by a polynomial in $z$.

(2) Every function $f$ analytic in a Jordan region $B$ and continuous on its closure $\bar{B}$ can be uniformly approximated on $\bar{B}$ by a polynomial in $z$.

The first is a generalization of Weierstrass' theorem, which requires arc $J$ to be a closed interval of the real axis. The second is a generalization of Runge's theorem, which requires $f$ to be analytic in $\bar{B}$. To prove this second theorem, Walsh approximated to $B$ by a sequence of Jordan regions $B_{n}$ with $\bar{B} \subset B_{n+1} \subset B_{n}$ for all $n$, and then applied Runge's theorem in $\bar{B}$ to the function $F_{n}(z)=f\left(\chi_{n}(z)\right)$, where $w=\chi_{n}(z)$ maps $B_{n}$ one-to-one conformally onto $B$. Walsh later extended this second theorem to sets $B$ which are the union of a finite number of disjoint Jordan regions. Thus he paved the way for the more comprehensive theorem proved later by Mergelyan: if $f$ is a function continuous on any closed bounded set $S$ and analytic at all interior points of $S$, then it can be approximated on $S$ uniformly by a polynomial in $z$.

Walsh maintained an active interest in interpolation, approximation and related topics over a period of about fifty years. Most recently he helped develop some of the fundamental theorems concerning spline interpolation and approximation both on the real line and in the complex plane. His contributions may be found in his published papers as well as in the monograph The theory of splines and their applications, which he wrote jointly with J. H. Ahlberg and E. N. Nilson.

In the related area of orthogonal expansions, special mention should be made of the so-called Walsh functions $\varphi_{n}^{(k)}(x)$. These are defined on the interval $0 \leqq x \leqq 1$ by the relations $\varphi_{0}(x) \equiv 1$;

$$
\begin{array}{cc}
\varphi_{1}^{(1)}(x)=1, \quad 0 \leqq x<\frac{1}{2} ; \quad \varphi_{1}^{(1)}(x)=-1, & \frac{1}{2}<x \leqq 1 ; \\
\varphi_{n+1}^{(2 k-1)}(x)=\varphi_{n+1}^{(2 k)}(x)=\varphi_{n}^{(k)}(2 x), & 0 \leqq x<\frac{1}{2} ; \\
\varphi_{n+1}^{(2 k-1)}(x)=-\varphi_{n+1}^{(2 k)}(x)=(-1)^{k+1} \varphi_{n}^{(k)}(2 x-1), & \frac{1}{2}<x \leqq 1 ; \\
k=1,2,3, \cdots, 2^{n-1} ; \quad n=1,2,3, \cdots .
\end{array}
$$

These functions, having some similarity to the Haar functions, were invented by Walsh in 1923 (see his paper 1923b). Now among the most widely used complete orthonormal systems, these functions serve as a valuable mathematical tool in communications engineering and other applied sciences. ${ }^{2}$ They are treated in Harmuth's book on Transmission of information by orthogonal functions, 2nd ed., Springer-Verlag, New York, 1972; in a survey article by Balashov and Rubinshtein, Series with

${ }^{2}$ Letter from T. J. Rivlin. 
respect to Walsh system and their generalization, J. Soviet Math. 1 (1973), 727-763 which lists 140 references; in an article by N. J. Fine, Encyclopaedic Dictionary of Physics, suppl. vol. 4, Pergamon, New York, 1971; and in Proc. Sympos. on Applications of Walsh Functions, 1970, 1971, 1972 and 1973. A fifth such symposium was held March 18-20, 1974 at the Catholic University of America in Washington, D.C.

Not only did Walsh himself contribute to the above described areas of research, but also he introduced many of his students to these areas. This is evident from the titles of the doctoral theses written under his direction.

Walsh had altogether thirty-one Ph.D. students. The present writer succeeded in contacting nearly all of them to ask for their recollections of him as a research advisor, teacher and friend. In what follows are recorded some of this memorabilia.

Nearly all his former students agreed that, as a thesis advisor, Walsh was patient, generous and considerate, and freely gave help and encouragement. This was true, not only during the developmental stages of each thesis, but even during the years afterwards. It must have given him a great deal of satisfaction that over the years so many of his students continued to contribute to mathematical literature, and that they wrote special papers in his honor on the occasion of his seventieth and seventy-fifth birthdays.

Their remembrance of Walsh as a teacher almost always includes certain rituals accompanying each of his lectures. The opening ritual was to fling the classroom windows wide open regardless of outside temperatures and to deliver his lecture pacing back and forth on the platform while the students might be freezing in their seats. The closing ritual seemed to have been to toss his chalk into the waste basket from whatever position he ended the lecture. Besides, during the run of a lecture he was occasionally known to have used an inattentive student as a target for the chalk. Elementary calculus and introductory complex function theory were his favorite courses, but whatever the subject he prepared his lectures with meticulous care and presented them in a deep, musical voice.

Nearly all his former students were impressed by his love of walking. Regardless of the weather he would hike the mile and a half (or so) from his home near Fresh Pond to Widener Library or Seaver Hall. When he accepted the appointment to the University of Maryland, his first prerequisite for a new home was that it be within walking distance of the University.

Some of his former students recollect his sense of humor and his enjoyment of practical jokes. For example, at the 1948 summer meetings of the Society in New Haven, a group photograph was being taken with the 
mathematicians seated on wide circular bleachers. As the camera rotated, Walsh got his companion to run with him from one end of the bleachers to the other and thus they appeared twice in the same composite photograph. ${ }^{3}$ As another instance, a former student who felt himself a novice in teaching sought pedagogic advice from Walsh, to which the latter gave the terse reply: "Always start writing in the upper left-hand corner of the blackboard".4.5

In summary, Walsh may be characterized as having had a strong sense of duty to religion, country and chosen work, as well as a love of art and music and a Thoreau-like love of nature. ${ }^{6}$ Above all, he was a resolute, hard worker, known to have spent long hours daily in his Widener study. He explored his problems thoroughly along all possible paths and byways, usually reporting his discoveries in a succession of papers. Often years later he revisited the same problems, searching for nuggets that he may have missed on his earlier explorations. When asked on one occasion how he managed to keep up the terrific pace of publication, Walsh pointed to the self-portrait of an artist showing Death standing nearby and the artist working to complete as much as possible in the little time that remained. ${ }^{3}$ The verdict of history will surely be that Walsh did win this race against time. He did leave a permanent imprint upon the mathematics of this century and especially upon the men and women whose mathematical careers he helped to launch.

In the words of the poet, Henry Wadsworth Longfellow, [Charles Sumner, stanza 9]

"So when a great man dies

For years beyond our ken

The light he leaves behind him lies

Upon the paths of men."

\section{PAPers By Joseph L. WaLSh}

1916 Note on Cauchy's integral formula, Ann. of Math. 18, 79-80.

1918 On the location of the roots of the Jacobian of two binary forms and of the derivative of a rational function, Trans. Amer. Math. Soc. 19, 291-298.

1920 a. On the proof of Cauchy's integral formula by means of Green's formula, Bull. Amer. Math. Soc. 26, 155-157.

${ }^{3}$ Letter from E. N. Nilson.

${ }^{4}$ Letter from T. J. Rivlin.

${ }^{5}$ Other anecdotes may be found in the two articles: D. V. Widder, Joseph Leonard Walsh, J. SIAM Numer. Anal. 3 (1966), 171-172; M. Marden, Homage to Walsh, J. Approximation Theory 5 (1972), ix-xiii.

- Letter from Maurice Heins. 
b. On the solution of linear equations in infinitely many variables by successive approximations, Amer. J. Math. 42, 91-96.

c. On the location of the roots of the derivative of a polynomial, Ann. of Math. 22, 128-144.

1921 a. On the location of the roots of the derivative of a polynomial, Comptes Rendus du Congrès International des Mathématiciens (Strasbourg, 1920), Publiés par Henri Villat, Toulouse, Edouard Privat, pp. 339-342.

b. On the location of the roots of the Jacobian of two binary forms and of the derivative of a rational function, Trans. Amer. Math. Soc. 22, 101-116.

c. A generalization of the Fourier cosine series, Trans. Amer. Math. Soc. 22, 230-239.

d. On the transformation of convex point sets, Ann. of Math. 22, 262-266.

e. Sur la position des racines des dérivées d'un polynome, C. R. Acad. Sci. Paris $172,662-664$.

f. Solution to proposed problem by Nathan Altschillercourt, "Find the surfaces all the plane sections of which are circles", Amer. Math. Monthly 28, 480.

g. (With N. Wiener), The equivalence of expansions in terms of orthogonal functions, J. Math. Phys. 1, 103-122

h. A theorem on cross-ratios in the geometry of inversion, Ann. of Math. 23, 45-51.

1922 a. A theorem on loci connected with cross-ratios, Rend. Circ. Mat. Palermo 46, 236-248.

b. On the location of the roots of the derivative of a polynomial, Proc. Nat. Acad. Sci. U.S.A. 8, 139-141.

c. A certain two-dimensional locus, Amer. Math. Monthly 29, 112-114.

d. Some two-dimensional loci connected with cross ratios, Trans. Amer. Math. Soc. 23, 67-88.

e. A generalization of normal congruences of circles, Bull. Amer. Math. Soc. 28, 456-462.

f. On the convergence of the Sturm-Liouville series, Ann. of Math. 24, 109-120.

g. On the location of the roots of certain types of polynomials, Trans. Amer. Math. Soc. 24, 163-180.

h. On the location of the roots of the Jacobian of two binary forms and of the derivative of a rational function, Trans. Amer. Math. Soc. 24, 31-69.

1923 a. Sur un théorème d'algèbre, C. R. Acad. Sci. Paris 176, 1361-1364.

b. A closed set of normal orthogonal functions, Amer. J. Math. 45, 5-24.

c. A property of Haar's system of orthogonal functions, Math. Ann. 90, 38-45.

1924 a. Sur la détermination d'une analytique par ses valeurs sur un contour, C. R. Acad. Sci. Paris 178, 58-60.

b. On the location of the roots of polynomials, Bull. Amer. Math. Soc. 30, 51-62.

c. Some two-dimensional loci, Quart. J. Pure Math. 1, 154-165.

d. On the expansion of analytic functions in series of polynomials, Trans. Amer. Math. Soc. 26, 155-170.

e. On the location of the roots of Lamé's polynomials, Tôhoku Math. J. 23, 312-317.

f. A generalization of evolutes, Rend. Circ. Mat. Palermo 48, 23-27.

g. (Book Review), The Strasbourg Congress, Bull. Amer. Math. Soc. 30, 461-464.

h. An inequality for the roots of an algebraic equation, Ann. of Math. 25, 285-286.

i. On Pellet's theorem concerning the roots of a polynomial, Ann. of Math. 26, 59-64. 
1925 a. Sur la position des racines des fonctions entières de genre zero et un, C. R. Acad. Sci. Paris 180, 2009-2011.

1926 a. Note on the location of the roots of a polynomial, Math. Z. 24, 733-742.

b. Uber die Entwicklung einer analytischen Funktion nach Polynomen, Math. Ann. 96, 430-436.

c. Uber die Entwicklung einer Funktion einer komplexen Veränderlichen nach Polynomen, Math. Ann. 96, 437-450.

d. Uber den Grad der Approximation einer analytischen Funktion, Sitzungsberichte Baverischen Akad. Wiss., 223-229.

1927 a. A paradox resulting from integeration by parts, Amer. Math. Monthly 34, 88.

b. On the expansion of harmonic functions in terms of harmonic polynomials, Proc. Nat. Acad. Sci. U.S.A. 13, 175-180.

c. On the degree of approximation to a harmonic function, Bull. Amer. Math. Soc. 33, 591-598.

1928 a. On the expansion of analytic functions in series of polynomials and in series of other analytic functions, Trans. Amer. Math. Soc. 30, 307-332.

b. (Book Review), The logarithmic potential, By G. C. Evans, Amer. Math. Monthly 35, 254-257.

c. On approximation to an arbitrary function of a complex variable by polynomials, Trans. Amer. Math. Soc. 30, 472-482.

d. Uber die Entwicklung einer harmonischen Funktion nach harmonischen Polynomen, J. Reine Angew. Math. 159, 197-209.

e. On the degree of approximation to an analytic function by means of rational functions, Trans. Amer. Math. Soc. 30, 838-847.

1929 a. Note on the expansion of analytic functions in series of polynomials and in series of other analytic functions, Trans. Amer. Math. Soc. 31, 53-57.

b. The approximation of harmonic functions by harmonic polynomials and by harmonic rational functions, Bull. Amer. Math. Soc. 35, 499-544.

c. On approximation by rational functions to an arbitrary function of a complex variable, Trans. Amer. Math. Soc. 31, 477-502.

d. Boundary values of an analytic function and the Tchebycheff method of approximation, Proc. Nat. Acad. Sci. U.S.A. 15, 799-802.

1930 a. On the overconvergence of sequences of polynomials of best approximation, Proc. Nat. Acad. Sci. U.S.A. 16, 297.

b. Boundary values of an analytic function and the Tchebycheff method of approximation, Trans. Amer. Math. Soc. 32, 335-390.

c. On the overconvergence of sequences of polynomials of best approximation, Trans. Amer. Math. Soc. 32, 794-816.

1931 a. Note on the overconvergence of sequences of polynomials of best approximation, Trans. Amer. Math. Soc. 33, 370-388.

b. The existence of rational functions of best approximation, Trans. Amer. Math. Soc. 33, 668-689.

c. On the overconvergence of certain sequences of rational functions of best approximation, Acta Math. 57, 411-435.

1932 a. On interpolation and approximation by rational functions with preassigned poles, Trans. Amer. Math. Soc. 34, 22-74.

b. An expansion of meromorphic functions, Proc. Nat. Acad. Sci. U.S.A. 18, 165-171.

c. On polynomial interpolation to analytic functions with singularities, Bull. Amer. Math. Soc. 38, 289-294. 
d. On the overconvergence of sequences of rational functions, Amer. J. Math. 54, 559-570.

e. On interpolation to harmonic functions by harmonic polynomials, Proc. Nat. Acad. Sci. U.S.A. 18, 514-517.

f. Interpolation and functions analytic interior to the unit circle, Trans. Amer. Math. Soc. 34, 523-556.

1933 a. Bateman on mathematical physics, Bull. Amer. Math. Soc. 39, 178-180. (Review).

b. Interpolation and an analogue of the Laurent development, Proc. Nat. Acad. Sci. U.S.A. 19, 203-207.

c. The Cauchy-Goursat theorem for rectifiable Jordan curves, Proc. Nat. Acad. Sci. U.S.A. 19, 540-541.

d. An extremal problem in analytic functions, Proc. Nat. Acad. Sci. U.S.A. 19, 900-902.

e. Note on polynomial interpolation to analytic functions, Proc. Nat. Acad. Sci. U.S.A. 19, 959-963.

f. Note on the location of the critical points of Green's function, Bull. Amer. Math. Soc. 39, 775-782.

g. A duality in interpolation to analytic functions by rational functions, Proc. Nat. Acad. Sci. U.S.A. 19, 1049-1053.

h. On series of interpolation and the degree of convergence of sequences of analytic functions, Tôhoku Math. J. 38, 375-389.

i. Note on the location of the roots of the derivative of a polynomial, Mathematica (Cluj) 8, 185-190.

1934 a. (With Helen G. Russell), On the convergence and overconvergence of sequences of polynomials of best simultaneous approximation to several functions analytic in distinct regions, Trans. Amer. Math. Soc. 36, 13-28.

b. On approximation to an analytic function by rational functions of best approximation, Math. Z. 38, 163-176.

c. Note on the orthogonality of Tchebycheff polynomials on confocal ellipses, Bull. Amer. Math. Soc. 40, 84-88.

d. Some interpolation series, Amer. Math. Monthly 41, 300-308.

e. Sur l'interpolation par fonctions rationnelles, C. R. Acad. Sci. Paris 198, 13771378.

f. Note on the location of the critical points of harmonic functions, Proc. Nat. Acad. Sci. U.S.A. 20, 551-554.

1935 a. Lemniscates and equipotential curves of Green's function, Amer. Math. Monthly 42, 1-17.

1936 a. A necessary condition for approximation by rational functions, Bull. Amer. Math. Soc. 42, 219-221.

b. The divergence of sequences of polynomials interpolating in roots of unity, Bull. Amer. Math. Soc. 42, 715-719.

c. Note on the behavior of a polynomial at infinity, Amer. Math. Monthly 43, 461-464.

d. A mean-value theorem for polynomials and harmonic polynomials, Bull. Amer. Math. Soc. 42, 923-930.

1937 a. Note on the curvature of level curves of Green's function, Proc. Nat. Acad. Sci. U.S.A. 23, 84-89.

b. (With W. E. Sewell), Note on degree of approximation to an integral by Riemann sums, Amer. Math. Monthly 44, 155-160. 
c. Note on the curvature of orthogonal trajectories of level curves of Green's function Proc. Nat. Acad. Sci. U.S.A. 23, 166-169.

d. On the shape of level curves of Green's function, Amer. Math. Monthly 44, 202-213.

e. Maximal convergence of sequences of harmonic polynomials, Ann. of Math. 38, 321-354.

f. (With G. M. Merriman), Note on the simultaneous orthogonality of harmonic polynomials on several curves, Duke Math. J. 3, 279-288.

g. (With W. E. Sewell), Note on the relation between continuity and degree of polynomial approximation in the complex domain, Bull. Amer. Math. Soc. 43, 557-563.

1938 a. Note on the curvature of orthogonal trajectories of level curves of Green's functions, Bull. Amer. Math. Soc. 44, 520-523.

b. (With W. Seidel), On the derivatives of functions analytic in the unit circle, Proc. Nat. Acad. Sci. U.S.A. 24, 337-340.

c. On interpolation and approximation by functions analytic and bounded in a given region, Proc. Nat. Acad. Sci. U.S.A. 24, 477-486.

d. (With W. E. Sewell), Note on degree of trigonometric and polynomial approximation to an analytic function, Bull. Amer. Math. Soc. 44, 865-873.

1939 a. (Book Review), Convergence, by W. L. Ferrar, Science 89, 59-60.

b. Note on the location of zeros of the derivative of a rational function whose zeros and poles are symmetric in a circle, Bull. Amer. Math. Soc. 45, 462470.

c. On interpolation by functions analytic and bounded in a given region, Trans. Amer. Math. Soc. 46, 46-65. MR 1, 10.

d. On the circles of curvature of the images of circles under a conformal map, Amer. Math. Monthly 46, 472-485. MR 1, 111.

1940 a. Note on the curvature of orthogonal trajectories of level curves of Green's function. III, Bull. Amer. Math. Soc. 46, 101-108. MR 1, 210.

b. On the degree of convergence of sequences of rational functions, Trans. Amer. Math. Soc. 47, 254-292. MR 1, 309.

c. Note on the degree of convergence of sequences of analytic functions, Trans. Amer. Math. Soc. 47, 293-304. MR 1, 310.

d. (With W. E. Sewell), Note on degree of trigonometric and polynomial approximation to an analytic function, in the sense of least pth powers, Bull. Amer. Math. Soc. 46, 312-319. MR 1, 309.

e. (With W. E. Sewell), Sufficient conditions for various degrees of approximation by polynomials, Duke Math. J. 6, 658-705. MR 2, 80.

1941 a. (Book Review), Sur les valeurs exceptionelles des fonctions méromorphes et de leurs dérivées, By Georges Valiron, Actualités Sci. Indust., no. 570, Hermann, Paris, 1937; Bull. Amer. Math. Soc. 47, 7-8.

b. (With W. E. Sewell), On the degree of polynomial approximation to analytic functions: problem $\beta$, Trans. Amer. Math. Soc. 49, 229-257. MR 2, 276.

c. (With W. Seidel), On approximation by euclidean and non-euclidean translations of an analytic function, Bull. Amer. Math. Soc. 47, 916-920. MR 4, 10.

1942 a. Note on the coefficients of overconvergent power series, Bull. Amer. Math. Soc. 48, 163-166. MR 3, 201.

b. (With W. Seidel), On the derivatives of functions analytic in the unit circle and their radii of univalence and of p-valence, Trans. Amer. Math. Soc. 52, 128-216. MR 4, 215. 
1943 a. Local civil time and date from time diagram, Proc. U.S. Naval Institute 69 (Whole No. 479), 23-24.

b. A new diagram for a universal small-area plotting sheet, Proc. U.S. Naval Institute 69 (Whole No. 487), 1221-1222.

1944 a. (With E. N. Nilson), Interpolation and approximation by functions analytic and bounded in a given region, Trans. Amer. Math. Soc. 55, 53-67. MR 5, 115.

1946 a. The running fix as used at sea (Some navigational wrinkles on "Pilot Chart of the North Atlantic Ocean," No. 1400), Hydrographic Office, Washington, D.C.

b. Note on the location of the critical points of harmonic functions, Bull. Amer. Math. Soc. 52, 346-347. MR 7, 382.

c. Overconvergence, degree of convergence, and zeros of sequences of analytic functions, Duke Math. J. 13, 195-234. MR 8, 201.

d. On degree of approximation on a Jordan curve to a function analytic interior to the curve by functions not necessarily analytic interior to the curve, Bull. Amer. Math. Soc. 52, 449-453. MR 7, 514.

e. Taylor's series and approximation to analytic functions, Bull. Amer. Math. Soc. 52, 572-579. MR 8, 144.

f. (Book Review), Table of arc $\sin x$ and Tables of associated Legendre functions, by Lyman J. Briggs, et al., Science 104, 41.

g. Note on the location of the zeros of the derivative of a rational function having prescribed symmetry, Proc. Nat. Acad. Sci. U.S.A. 32, 235-237. MR 8, 144.

1947 a. A rigorous treatment of the first maximum problem in the calculus, Amer. Math. Monthly 54, 35-36.

b. On the location of the critical points of harmonic measure, Proc. Nat. Acad. Sci. U.S.A. 33, 18-20. MR 8, 461.

c. (With E. N. Nilson), Note on the degree of convergence of sequences of polynomials, Bull. Amer. Math. Soc. 53, 116-117. MR 9, 23.

d. Note on the derivatives of functions analytic in the unit circle, Bull. Amer. Math. Soc. 53, 515-523. MR 9, 23.

e. Note on the critical points harmonic functions, Proc. Nat. Acad. Sci. U.S.A. 33, 54-59. MR 8, 513.

f. The location of the critical points of simply and doubly periodic functions, Duke Math. J. 14, 575-586. MR 9, 180.

1948 a. On the critical points of functions possessing central symmetry on the sphere, Amer. J. Math. 70, 11-21. MR 9, 428.

b. Note on the location of the critical points of harmonic functions, Bull. Amer. Math. Soc. 54, 191-195. MR 9, 432.

c. The critical points of linear combinations of harmonic functions, Bull. Amer. Math. Soc. 54, 196-205. MR 9, 432.

d. Critical points of harmonic functions as positions of equilibrium in a field of force, Proc. Nat. Acad. Sci. U.S.A. 34, 111-119. MR 9, 432.

e. Methods of symmetry and critical points of harmonic functions, Proc. Nat. Acad. Sci. U.S.A. 34, 267-271. MR 9, 585.

f. On the location of the zeros of the derivatives of a polynomial symmetric in the origin, Bull. Amer. Math. Soc. 54, 942-945. MR 10, 250.

1949 a. (With W. E. Sewell and H. M. Elliott), On the degree of convergence of harmonic polynomials to harmonic functions, Proc. Nat. Acad. Sci. U.S.A. 35, 59-62. MR 10, 374.

b. (With E. N. Nilson), On functions analytic in a region: Approximation in the sense of least pth powers, Trans. Amer. Math. Soc. 65, 239-258. MR 10, 524. 
c. (With A. S. Galbraith and W. Seidel), On the growth of derivatives of functions omitting two values, Trans. Amer. Math. Soc. 67, 320-326. MR 11, 344.

d. (With W. E. Sewell and H. M. Elliott), On the degree of polynomial approximation to harmonic and analytic functions, Trans. Amer. Math. Soc. 67, 381-420. MR 11, 515.

e. (With W. E. Sewell), On interpolation to an analytic function in equidistant points: Problem $\beta$, Bull. Amer. Math. Soc. 55, 1177-1180. MR 11, 344.

1950 a. On distortion at the boundary of a conforn:al map, Proc. Nat. Acad. Sci. U.S.A. 36, 152-156. MR 11, 507.

b. (With H. Margaret Elliott), Polynomial approximation to harmonic and analytic functions: generalized continuity conditions, Trans. Amer. Math. Soc. 68, 183-203. MR 11, 515.

c. The location of critical points of harmonic functions, Leopoldo Fejér et Frederico Riesz LXX Annos Natis Dedicatus, Pars B. Acta Sci. Math. Szeged 12, 61-65. MR 12, 26.

d. (With H. G. Russell), On simultaneous interpolation and approximation by functions analytic in a given region, Trans. Amer. Math. Soc. 69, 416-439. MR $12,813$.

1951 a. On Rouche's theorem and the integral-square measure of approximation, Proc. Amer. Math. Soc. 2, 671-681. MR 13, 335.

b. Note on the location of the critical points of a real rational function, Proc. Amer. Math. Soc. 2, 682-685. MR 13, 451.

c. Note on approximation by bounded analytic functions, Proc. Nat. Acad. Sci. U.S.A. 37, 821-826. MR 13, 545.

1952 a. On Rouche's theorem and the integral-square measure of approximation, Proc. Internat. Congress Math., vol. 1, Amer. Math. Soc., Providence, R.I., pp. 405406.

b. (With E. N. Nilson), Note on overconvergence in sequences of analytic functions, Proc. Amer. Math. Soc. 3, 442-443. MR 13, 927.

c. Polynomial expansions of functions defined by Cauchy's integral, J. Math. Pures Appl. (9) 31, 221-244. MR 14, 547.

d. Note on the location of zeros of extremal polynamials in the non-euclidean plane, Acad. Serbe Sci. Publ. Inst. Math. 4, 157-160. MR 14, 164.

e. (With Philip Davis), Interpolation and orthonormal systems, J. Analyse Math. 2, 1-28. MR 16, 580.

f. Degree of approximation to functions on a Jordan curve, Trans. Amer. Math. Soc. 73, 447-458. MR 14, 630.

g. (With H. Margaret Elliott), Degree of approximation on a Jordan curve, Proc. Nat. Acad. Sci. U.S.A. 38, 1058-1066. MR 14, 741.

1953 a. An interpolation series expansion for a meromorphic function, Trans. Amer. Math. Soc. 74, 1-9. MR 14, 741.

b. On continuity properties of derivatives of sequences of functions, Proc. Amer. Math. Soc. 4, 69-75. MR 14, 736; 1278.

c. (With T. S. Motzkin), On the derivative of a polynomial and Chebyshev approximation, Proc. Amer. Math. Soc. 4, 76-87. MR 15, 701.

d. Note on the shape of level curves of Green's function, Duke Math. J. 20, 611-615. MR 15, 310.

e. Note on the shape of level curves of Green's function, Amer. Math. Monthly 60, 671-674. MR 15, 424. 
f. (With David Young), On the accuracy of the numerical solution of the Dirichlet problem by finite differences, J. Res. Nat. Bur. Standards 51, 343-363. MR 15, 562.

1954 a. An interpolation problem for harmonic functions, Amer. J. Math. 76, 259-272. MR 16, 588.

b. (With David Young), On the degree of convergence of solutions of difference equations to the solution of the Dirichlet problem, J. Math. Phys. 33, 80-93. MR 15, 746.

c. (With Philip Davis), On representations and extensions of bounded linear functionals defined on classes of analytic functions, Trans. Amer. Math. Soc. 76, 190-206. MR 15, 803.

d. (With J. P. Evans), Note on the distribution of zeros of extremal polynomials, Proc. Nat. Acad. Sci. U.S.A. 40, 332-337. MR 15, 954.

e. (With J. P. Evans), On approximation by bounded analytic functions, Arch. Math. 5, 191-196. MR 15, 947.

f. Sur l'approximation par fonctions analytiques bornées, C. R. Acad. Sci. Paris 239, 1339-1341. MR 16, 811.

g. Sur la représentation conforme des aires multiplement connexes, C. R. Acad. Sci. Paris 239, 1572-1574. MR 16, 581.

h. Sur la représentation conforme des aires multiplement connexes, C. R. Acad. Sci. Paris 239, 1756-1758. MR 16, 811.

i. (With M. Fekete), On the asymptotic behavior of polynomials with extremal properties, and of their zeros, J. Analyse Math. 4, 49-87. MR 17, 354.

j. Détermination d'une fonction analytique par ses valeurs données dans une infinité dénombrable de points, Bull. Soc. Math. Belg., 52-70. MR 17, 601.

1955 a. (With D. Gaier), Zur Methode der variablen Gebiete bei der Randverzerrung, Arch. Math. 6, 77-86. MR 16, 348.

b. (With T. S. Motzkin), Least pth power polynomials on a real finite point set, Trans. Amer. Math. Soc. 78, 67-81. MR 16, 585.

c. A generalization of Jensen's theorem on the zeros of the derivative of a polynomial, Amer. Math. Monthly 62, 91-93. MR 16, 818.

d. (With J. P. Evans), On interpolation to a given analytic function by analytic functions of minimum norm, Trans. Amer. Math. Soc. 79, 158-172. MR 16, 1011.

e. Sur l'approximation par fonctions rationnelles et par fonctions holomorphes bornées, Ann. Mat. Pura Appl. (4) 39, 267-277. MR 17, 1077.

1956 a. (With Mishael Zedek), On generalized Tchebycheff polynomials, Proc. Nat. Acad. Sci. U.S.A. 42, 99-104. MR 17, 730.

b. (With L. Rosenfeld), On the boundary behavior of a conformal map, Trans. Amer. Math. Soc. 81, 49-73. MR 17, 836.

c. Best-approximation polynomials of given degree, Proc. Sympos. Appl. Math., Vol. VI, Numerical Analysis, Santa Monica, Aug. 1953, Amer. Math. Soc., Providence, R.I., pp. 213-218. MR 18, 32.

d. On the conformal mapping of multiply connected regions, Trans. Amer. Math. Soc. 82, 128-146. MR 18, 290.

e. (With T. S. Motzkin), Least pth power polynomials on a finite point set, Trans. Amer. Math. Soc. 83, 371-396. MR 18, 479.

f. (With J. P. Evans), On the location of the zeros of certain orthogonal functions, Proc. Amer. Math. Soc. 7, 1085-1090. MR 18, 725.

g. Note on degree of approximation to analytic functions by rational functions with preassigned poles, Proc. Nat. Acad. Sci. U.S.A. 42, 927-930. MR 18, 569. 
h. "Birkhoff, George David", article in Encyclopedia Britannica.

i. (With M. Fekete), On restricted infrapolynomials, J. Analyse Math. 5, 47-76. MR 19, 263.

1957 a. (With T. S. Motzkin), Underpolynomials and infrapolynomials, Illinois J. Math. 1, 406-426. MR 19, 643.

b. (With T. S. Motzkin), Polynomials of best approximation on a real finite point set, Proc. Nat. Acad. Sci. U.S.A. 43, 845-846. MR 19, 852.

c. (With M. Fekete), Asymptotic behavior of restricted extremal polynomials and of their zeros, Pacific J. Math. 7, 1037-1064. MR 19, 1045.

d. (With David Young), Lipschitz conditions for harmonic and discrete harmonic functions, J. Math. Phys. 36, 138-150. MR 20, \#2532.

1958 a. On approximation by bounded analytic functions, Trans. Amer. Math. Soc. 87, 467-484. MR 20 \#3298.

b. A generalization of Faber's polynomials, Math. Ann. 136, 23-33. MR 21 \#725.

c. Complex numbers and complex variables, Laplace's differential equation, and Conformal mapping, three articles in the McGraw-Hill Encyclopedia of Science and Technology.

d. Approximation by bounded analytic functions, Seminars on Analytic Functions, vol. II, pp. 73-87, published by U.S. Air Force, Office of Scientific Research, Washington, D.C.

e. On infrapolynomials with prescribed constant term, J. Math. Pures Appl. (9) 37, 295-316. MR 20 \#7098.

1959 a. On extremal approximations, On Numerical Approximation, Univ. of Wisconsin Press, Madison, Wis., pp. 209-216. MR 21 \#421.

b. (With T. S. Motzkin), Location of zeros of infrapolynomials, Compositio Math. 14, 50-70. MR 21 \#3539.

c. Approximation on a line segment by bounded analytic functions: Problem $\beta$, Proc. Amer. Math. Soc. 10, 270-272. MR 21 \#6443a.

d. Note on least-square approximation to an analytic function by polynomials, as measured by a surface integral, Proc. Amer. Math. Soc. 10, 273-279. MR 23 \#A1047.

e. Approximation by bounded analytic functions: General configurations, Proc. Amer. Math. Soc. 10, 280-285. MR 21 \#6443b.

f. (With T. S. Motzkin), Polynomials of best approximation on a real finite point set. I, Trans. Amer. Math. Soc. 91, 231-245. MR 21 \#7388.

g. (With H. G. Russell), Integrated continuity conditions and degree of approximation by polynomials or by bounded analytic functions, Trans. Amer. Math. Soc. 92, 355-370. MR 21 \#7311.

h. Note on approximation by bounded analytic functions (Problem $\alpha$ ), Math. Z. 72, 47-52. MR 22 \#776.

i. (With T. S. Motzkin), Polynomials of best approximation on an interval, Proc. Nat. Acad. Sci. U.S.A. 45, 1523-1528. MR 22 \#9773.

j. Note on invariance of degree of polynomial and trigonometric approximation under change of independent variable, Proc. Nat. Acad. Sci. U.S.A. 45, 15281533. MR 23 \#A1191.

k. (With H. J. Landau), On canonical conformal maps of multiply connected regions, Trans. Amer. Math. Soc. 93, 81-96. MR 28 \#4093.

1. The analogue for maximally convergent polynomials of Jentzsch's theorem, Duke Math. J. 26, 605-616. 
1960 a. Solution of the Dirichlet problem for the ellipse by interpolating harmonic polynomials, J. Math. Mech. 9, 193-196. MR 22 \#4891.

b. On the asymptotic properties of extremal polynomials with prescribed constant term, Math. Z. 73, 339-345. MR 22 \#2715.

c. Note on polynomial approximation on a Jordan arc, Proc. Nat. Acad. Sci. U.S.A. 46, 981-983. MR 22 \#12338.

d. On degree of approximation by bounded harmonic functions, J. Math. Pures Appl. (9) 39, 201-220. MR 27 \#1609.

e. (With T. S. Motzkin), Best approximators within a linear family on an interval, Proc. Nat. Acad. Sci. U.S.A. 46, 1225-1233. MR 28 \#5279.

f. Degree of approximation by bounded harmonic functions, Proc. Nat. Acad. Sci. U.S.A. 46, 1390-1393. MR 22 \#12338b.

g. Note on degree of approximation by bounded analytic functions: Problem $\beta$, Trans. Amer. Math. Soc. 96, 246-258. MR 22 \#11140.

1961 a. The circles of curvature of the curves of steepest descent of Green's function, Amer. Math. Monthly 68, 323-329. MR 23 \#A3272.

b. (With T. S. Motzkin), Conformal maps of small disks, Proc. Nat. Acad. Sci. U.S.A. 47, 1838-1843. MR 26 \#307.

c. (With $\mathrm{O}$. Shisha), The zeros of infrapolynomials with some prescribed coefficients, J. Analyse Math. 9, 111-160. MR 25 \#174.

d. (With J. P. Evans), Approximation by bounded analytic functions to functions represented by Dirichlet series, Proc. Amer. Math. Soc. 12, 875-879. MR 25 \#4109.

e. A new generalization of Jensen's theorem on the zeros of the derivative of a polynomial, Amer. Math. Monthly 68, 978-983. MR 24 \#A2009.

1962 a. Degree of polynomial approximation to an analytic function as measured by a surface integral, Proc. Nat. Acad. Sci. U.S.A. 48, 26-32. MR 24 \#A2176.

b. (With J. H. Ahlberg and E. N. Nilson), Best approximation properties of the spline fit, J. Math. Mech. 11, 225-234. MR 25 \#738.

c. Asymptotic properties of polynomials with auxiliary conditions of interpolation, Ann. Polon. Math. 12, 17-24. MR 27 \#1605.

d. (With T. S. Motzkin), Polynomials of best approximation on an interval. II, Proc. Nat. Acad. Sci. U.S.A. 48, 1533-1537. MR 26 \#2788.

e. On the convexity of the ovals of lemniscates, Studies in Mathematical Analysis and Related Topics, Stanford University Press, Stanford, Calif., pp. 419-423. MR 27 \#1606.

f. Approximation par les fonctions holomorphes bornées. Probleme $\beta^{\prime}$, J. Math. Pures Appl. (9) 41, 213-232. MR 27 \#5914.

1963 a. (With T. S. Motzkin), Zeros of the error function for Tchebycheff approximation in a small region, Proc. London Math. Soc. (3) 13, 90-98. MR 26 \#1667.

b. Restricted infrapolynomials and trigonometric infrapolynomials, Proc. Nat. Acad. Sci. U.S.A. 49, 302-304. MR 27 \#2613.

c. A generalization of Fejér's principle concerning the zeros of extremal polynomials, Proc. Amer. Math. Soc. 14, 44-51. MR 27 \#271.

d. A sequence of rational functions with application to approximation by bounded analytic functions, Duke Math. J. 30, 177-189. MR 30 \#2155.

e. (With $\mathrm{O}$. Shisha), The zeros of infrapolynomials with prescribed values at given points, Proc. Amer. Math. Soc. 14, 839-844. MR 27 \#3785.

f. Note on the convergence of approximating rational functions of prescribed type, Proc. Nat. Acad. Sci. U.S.A. 50, 791-794. MR 28 \#400; 28 \#1247.

g. (Book Review), Analytic function theory, by E. Hille, SIAM Rev. 5, 377-378. 
1964 a. Padé approximants as limits of rational functions of best approximation, J. Math. Mech. 13, 305-312. MR 28 \#4283.

b. (With O. Shisha), On the location of the zeros of some infrapolynomials with prescribed coefficients, Pacific J. Math. 14, 1103-1109. MR 30 \#232.

c. (With $\mathrm{O}$. Shisha), Extremal polynomials and the zeros of the derivative of a rational function, Proc. Amer. Math. Soc. 15, 753-758. MR 29 \#4876.

d. The convergence of sequences of rational functions of best approximation, Math. Ann. 155, 252-264. MR 29 \#1484.

e. A theorem of Grace on the zeros of polynomials, revisited, Proc. Amer. Math. Soc. 15, 354-360. MR 28 \#4092.

f. (With Z. Rubinstein), On the location of the zeros of a polynomial whose center of gravity is given, J. Analyse Math. 12, 129-142. MR 29 \#4877.

g. (With A. Sharma), Least squares and interpolation in roots of unity, Pacific J. Math. 14, 727-730. MR 28 \#5278.

h. Surplus free poles of approximating rational functions, Proc. Nat. Acad. Sci. U.S.A. 52, 896-901. MR 30 \#3983.

i. (With Maynard Thompson), Approximation with auxiliary conditions, J. Math. Mech. 13, 1015-1019. MR 30 \#1253.

$\mathrm{j}$. The location of the zeros of the derivative of a rational function, revisited, J. Math Pures Appl. (9) 43, 353-370. MR 31 \#3582.

1965 a. Geometry of the zeros of the sums of linear fractions, Trans. Amer. Math. Soc. 114, 30-39. MR 31 \#3579.

b. (With J. H. Ahlberg and E. N. Nilson), Fundamental properties of generalized splines, Proc. Nat. Acad. Sci. U.S.A. 52, 1412-1419. MR 36 \#6846.

c. (With J. H. Ahlberg and E. N. Nilson), Best approximation and convergence properties of higher-order spline approximations, J. Math. Mech. 14, 231-243. MR 35 \#5823.

d. (With A. Sinclair), On the degree of convergence of extremal polynomials and other extremal functions, Trans. Amer. Math. Soc. 115, 145-160. MR 33 \#7564.

e. The convergence of sequences of rational functions of best approximation. II, Trans. Amer. Math. Soc. 116, 227-237. MR 32 \#6120.

f. (With J. H. Ahlberg and E. N. Nilson), Extremal, orthogonality, and convergence properties of multidimensional splines, J. Math. Anal. Appl. 12, 27-48. MR 37 \#661.

g. (With J. H. Ahlberg and E. N. Nilson), Convergence properties of generalized splines, Proc. Nat. Acad. Sci. U.S.A. 54, 344-350. MR 36 \#6847.

h. Hyperbolic capacity and interpolating rational functions, Duke Math. J. 32, 369-379. MR 31 \#6081.

i. The convergence of sequences of rational functions of best approximation with some free poles, Approximation of Functions (Proc. Sympos. General Motors Res. Lab., 1964), Henry L. Garabedian, Ed., Elsevier, Amsterdam, 1965, pp. 1-16. MR 32 \#4441.

1966 a. (With T. S. Motzkin), Mean approximation on an interval for an exponent less than one, Trans. Amer. Math. Soc. 122, 443-460. MR 34 \#1769.

b. Approximation by polynomials: Uniform convergence as implied by mean convergence, Proc. Nat. Acad. Sci. U.S.A. 55, 20-25. MR 32 \#5891.

c. Approximation by polynomials: Uniform convergence as implied by mean convergence, II. Proc. Nat. Acad. Sci. U.S.A. 55, 1405-1407. MR 35 \#4443.

d. (With H. G. Russell), Hyperbolic capacity and interpolating rational functions. II, Duke Math. J. 33, 275-279. MR 33 \#1624. 
e. The convergence of approximating rational functions of prescribed type, Contemporary Problems in the Theory of Analytic Functions (M. A. Lavrent'ev, Ed.), Proc. Internat. Conference on the Theory of Analytic Functions (Erevan, 1965), "Nauka”, Moscow, 1966, pp. 304-308. (Russian). MR 35 \#3069.

f. Approximation by polynomials: Uniform convergence as implied by mean convergence. III, Proc. Nat. Acad. Sci. U.S.A. 56, 1406-1408. MR 35 \#4444.

1967 a. Best approximation by rational functions and by meromorphic functions with some free poles, J. Analyse Math. 18, 359-375. MR 36 \#1673.

b. On the convergence of sequences of rational functions, SIAM J. Numer. Anal. 4, 211-221. MR 36 \#1675.

c. An extension of the generalized Bernstein lemma, Colloq. Math. 16, 91-92. MR 35 \#6844.

d. (With J. H. Ahlberg and E. N. Nilson), Complex cubic splines, Trans. Amer. Math. Soc. 129, 391-413. MR 36 \#573.

1968 a. Degree of approximation by rational functions and polynomials, Michigan Math. J. 15, 109-110. MR 36 \#6845.

b. Note on classes of functions defined by integrated Lipschitz conditions, Bull. Amer. Math. Soc. 74, 344-346. MR 36 \#2807.

c. (With T. S. Motzkin), A persistent local maximum of the pth power deviation on an interval, $p<1$, Pacific J. Math. 24, 133-142. MR 38 \#4868.

d. The convergence of sequences of rational functions of best approximation. III, Trans. Amer. Math. Soc. 130, 167-183. MR 36 \#1674.

e. Approximation by bounded analytic functions: Uniform convergence as implied by mean convergence, Trans. Amer. Math. Soc. 130, 406-413. MR 36 \#3997.

f. (With J. H. Ahlberg and E. N. Nilson), Cubic splines on the real line, J. Approximation Theory 1, no. 1, 5-10. MR 37 \#6650.

1969 a. (With E. B. Saff), Extensions of D. Jackson's theorem on best complex polynomial mean approximation, Trans. Amer. Math. Soc. 138, 61-69. MR 39 \#3001.

b. Inequalities expressing degree of convergence of rational functions, J. Approximation Theory 2, 160-166. MR 39 \#7115.

c. (With J. H. Ahlberg and E. N. Nilson), Properties of analytic splines. I. Complex polynominal splines, J. Math. Anal. Appl. 27, 262-278. MR 42 \#8136.

d. Note on approximation by bounded analytic functions, Problem $\alpha$ : General configurations, Aequationes Math. 3, 160-164. MR 41 \#5632.

e. Approximations to a function by a polynomial in a given function, Amer. Math. Monthly 76, 1049-1050.

f. (With Z. Rubinstein), Extensions and some applications of the coincidence theorems, Trans. Amer. Math. Soc. 146, 413-427. MR 40 \#4428.

1970 a. (With W. J. Schneider), On the shape of the level loci of harmonic measure, J. Analyse Math. 23, 441-460. MR 42 \#6205.

b. Approximation by rational functions: Open problems, J. Approximation Theory 3, 236-242. MR 43 \#538.

c. Note on degree of convergence of sequences of rational functions of prescribed type, Proc. Nat. Acad. Sci. U.S.A. 67, 1188-1191. MR 42 \#4748.

1971 a. (With J. H. Ahlberg and E. N. Nilson), Complex polynomial splines on the unit circle, J. Math. Anal. Appl. 33, 234-257. MR 43 \#3696.

b. (With Dov Aharonov), Some examples in degree of approximation by rational functions, Trans. Amer. Math. Soc. 159, 427-444. MR 44 \#6974. 
c. Mean approximation by polynomials on a Jordan curve, J. Approximation Theory 4, 263-268. MR 45 \#3726.

1972 a. (With Dov Aharonov), On the convergence of rational functions of best approximation to a meromorphic function, J. Math. Anal. Appl. 40, 418-426. MR 47 \#5263.

b. (With Myron Goldstein), Approximation by rational functions on Riemann surfaces, Proc. Amer. Math. Soc. 36, 464-466. MR 47 \#2072.

c. Note on the convergence of sequences of rational functions, Proc. Nat. Acad. Sci. U.S.A. 69, 2963-2964. MR 46 \#7522.

1973 a. (With T. S. Motzkin), Equilibrium of inverse distance forces in three dimensions, Pacific J. Math. 44, 241-250. MR 47 \#5234.

b. (With E. B. Saff), On the convergence of rational functions which interpolate in the roots of unity, Pacific $\mathrm{J}$. Math. 45, 639-641.

c. History of the Riemann mapping theorem, Amer. Math. Monthly 80, 270-276.

1974 a. The role of the pole in rational approximation, Rocky Mountain Math. J. (to appear).

b. (With P. M. Gauthier and Alice Roth), Uniform approximation in the spherical metric (in preparation).

\section{BOoKS BY Joseph L. WALSH}

1. Approximation by Polynomials in the Complex Domain, Mémorial des Sciences Mathématiques, Gauthier-Villars, Paris, 1935, ii +72 pp.

2. Interpolation and Approximation by Rational Functions in the Complex Domain, Colloquium Publications, Vol. 20, American Mathematical Society, Providence, R.I., 1935, ix +382 pp.; 2nd edition, 1952; 3rd edition, 1960; 4th rev. edition, 1965; 5th edition, 1969; Russian translation, IL, Moscow, 1961. MR 36 \#1671; 1672b, c.

3. A Bibliography on Orthogonal Polynomials (with J. A. Shohat and Einar Hille), National Research Council, Bulletin No. 103, Washington, D.C., 1940, ix +204 pp.

4. The Location of Critical Points of Analytic and Harmonic Functions, Colloquium Publications, Vol. 34, American Mathematical Society, Providence, R.I., 1950, viii +384 pp. MR 12, 249.

5. Approximation by Bounded Analytic Functions, Mémorial des Sciences Mathématiques, Fasc. 144, Gauthier-Villars, Paris, 1960, 66 pp. MR 22 \#9770.

6. A Rigorous Treatment of Maximum-Minimum Problems in the Calculus, D. C. Heath, Boston, Mass., 1962, 22 pp.

7. The Theory of Splines and Their Applications, (with J. H. Ahlberg and E. N. Nilson), Academic Press, New York and London, 1967, xi+284 pp. MR 39 \#684.

\section{Walsh's Ph.D. Students}

(With journal reference to some or all their thesis results)

1928 MORRIS MARDEN, On the location of the roots of the jacobian of two binary forms and of the derivative of a rational function, Trans. Amer. Math. Soc. 32 (1930), 81-109.

1930 ORIN J. FARRELL, On the expansion of harmonic functions in series of harmonic polynomials belonging to a simply connected region, Amer. J. Math. 57 (1935).

1931 CECIL T. HOLMES, Approximation of harmonic functions in 3 dimensions by harmonic polynomials (not published). 
1932 JOSEPH L. DOOB, Boundary values of analytic functions, Trans. Amer. Math. Soc. 34 (1932), 153-170; ibid 35 (1933), 418-451; On a theorem of Gross and Iversen, Ann. of Math. 33 (1932), 753-757.

1932 HELEN G. RUSSELL, On the convergence and overconvergence of sequences of polynomials of best simultaneous approximation to several functions analytic in distinct regions, Trans. Amer. Math. Soc. 36 (1934), 13-28.

1935 JOHN H. CURTISS, Interpolation in regularly distributed points, Trans. Amer. Math. Soc. 38 (1935), 458-473; On the Jacobi series, Trans. Amer. Math. Soc. 49 (1941), 467-501. MR 2, 355.

1935 YU-CHENG SHEN, On interpolation and approximation by rational functions with preassigned poles, J. Chinese Math. Soc. 1 (1936), 154-173.

1936 WALTER E. SEWELL, Generalized derivatives and approximation by polynomials, Trans. Amer. Math. Soc. 41 (1937), 84-120.

1937 ZEHMAN I. MOSESSON, Maximal sequences of polynomials (not published).

1938 FLOYD E. ULRICH, Problem of type for a certain class of Riemann surfaces, Duke Math. J. 5 (1939), 567-589. MR 1, 8.

1940 MAURICE H. HEINS, Extremal problems for functions analytic and singlevalued in a doubly-connected region, Amer. J. Math. 62 (1940), 91-106. MR 1, 114; On the iteration of functions which are analytic and single-valued in a given multiply-connected region, Amer. J. Math. 63 (1941), 461-480. MR 2, 275.

1940 ABRAHAM SPITZBART, Approximation in the sense of least pth powers with a single auxiliary condition of interpolation, Bull. Amer. Math. Soc. 52 (1946), 338-346. MR 7, 425.

1941 EDWIN N. NILSON, Interpolation and approximation of analytic functions by functions analytic and bounded in a given region; Interpolation and approximation by functions analytic and bounded in a given region; (published with J. L. Walsh), Trans. Amer. Math. Soc. 55 (1944), 53-67; MR 5, 115; ibid. 65 (1949), 239258. MR 10, 524.

1942 LYNN H. LOOMIS, The radius and modulus of $n$-valence for analytic functions where first $n-1$ derivatives vanish at a point, Bull. Amer. Math. Soc. 46 (1940), 496-501. MR 1, 308; On an inequality of Seidel and Walsh, 48 (1942), 908-911. MR 5, 37.

1947 IVAN R. HERSHNER, Jr., Radii of univalence and p-valence of functions analytic in the unit circle (not published).

1948 H. MARGARET ELLIOTT, On the degree of approximation to harmonic functions by harmonic polynomials, Trans. Amer. Math. Soc. 67 (1949), 381-420.

1949 HELLEN KELSALL NICKERSON, Studies in overconvergence, Bull. Amer. Math. Soc. 55 (1949), 1061.

1952 ALAN F. KAY, Distribution of zeros of sequences of polynomials of unbounded degree, Proc. Amer. Math. Soc. 6 (1955), 571-582. MR 17, 247.

1952 ISAAC E. BLOCK, Kernel functions and class $L^{2}$, Proc. Amer. Math. Soc. 4 (1953), 110-117. MR 14, 989; Duke Math. J. 19 (1952), 367-378. MR 14, 153.

1953 THEODORE J. RIVLIN, On sufficient conditions for overconvergence, Proc. Amer. Math. Soc. 6 (1955), 597-602. MR 17, 138.

1953 LAWRENCE ROSENFELD, On the boundary of a conformal map, Trans. Amer. Math. Soc. 81 (1956), 49-73.

1953 JACQUELINE P. EVANS, On approximation and interpolation by functions analytic in a given region and an application to orthonormal systems, Arch. Math. 5 (1954), 191-196; Proc. Nat. Acad. Sci. U.S.A. 40 (1954), 332-337; Trans. Amer. Math. Soc. 79 (1955), 158-172; Proc. Amer. Math. Soc. 7 (1956), 1085-1090. 
1954 RICHARD S. VARGA, Semi-infinite and infinite strips of zeros, Univ. e Politecnio Torino, Rend. Sem. Math. 11 (1951/52), 289-296. MR 14 \#546.

1956 MISHAEL ZEDEK, On generalized Tchebycheff polynomials, Proc. Nat. Acad. Sci. U.S.A. 42 (1956), 99-104.

1957 HENRY J. LANDAU, On canonical conformal maps of multiply connected domains, Trans. Amer. Math. Soc. 99 (1961), 1-20. MR 22 \#12212.

1961 VINCENT C. WILLIAMS, On conformal maps of regions of infinite connectivity, Trans. Amer. Math. Soc. 155 (1971), 427-453.

1962 DOROTHY B. SHAFFER, Shape of level loci of Green's function and other harmonic functions, J. Analyse Math. 17 (1966), 59-70; J. Math. Mech. 19 (1969), 41-48.

1963 VICTOR M. MANJARREZ, Polynomial bases for compact sets in the plane, Trans. Amer. Math. Soc. 132 (1968), 541-551.

1964 JERRY L. FIELDS, Rational approximations to hypergeometric functions, Math. Comp. 19 (1965), 606-624.

1968 EDWARD B. SAFF, Interpolation and functions of class $H(k, \alpha, 2)$, J. Approximation Theory 1 (1968), 488-492. MR 39 \#2992; Trans. Amer. Math. Soc. 138 (1969), 61-69; ibid 141 (1969), 79-92. MR 39 \#4406.

1973 MARVIN E. ORTEL, Approximation by bounded analytic functions.

Current address: Department of Mathematics, University of Wisconsin, Milwaukee, Wisconsin 53201 